(C) [2005] IEEE. Reprinted, with permission, from [L. Wang, S.W. Su, B.G. Celler and E. Ambikairajah, Analysis of Orientation Error of Triaxial Accelerometers on the Assessment of Energy Expenditure, Engineering in Medicine and Biology Society, 2005. IEEE-EMBS 2005. 27th Annual International Conference of the, 17-18 Jan. 2006]. This material is posted here with permission of the IEEE. Such ermission of the IEEE does not in any way imply IEEE endorsement of any of the University of Technology, Sydney's products or services. Internal or personal use of this material is permitted. However, permission to reprint/republish this material for advertising or promotional purposes or for creating new collective works for resale or redistribution must be obtained from the IEEE by writing to pubspermissions@ieee.org. By choosing to view this document, you agree to all provisions of the copyright laws protecting it 


\title{
Analysis of Orientation Error of Triaxial Accelerometers on the Assessment of Energy Expenditure
}

\author{
L. Wang, S.W. Su, B.G. Celler and E. Ambikairajah \\ Human Performance Lab, School of Electrical Engineering \& Telecommunications \\ University of New South Wales, UNSW Sydney N.S.W. 2052 Australia \\ Email: luwang@student.unsw.edu.au,steven.su@unsw.edu.au,b.celler@unsw.edu.au, and ambi@ee.unsw.edu.au
}

\begin{abstract}
This paper investigates the effects of orientation error in the positioning of triaxial accelerometers on the assessment of energy expenditure. Four subjects walked on a treadmill at varying velocities ranging from $4 \mathrm{~km} . \mathrm{h}^{-1}$ to $5 \mathrm{~km} \cdot \mathrm{h}^{-1}$. During each test, a triaxial accelerometer attached to the lower back at arbitrary orientations to record body accelerations. Energy expenditure was estimated by the sum of the integrals of the absolute value of accelerometer output from all the three measurement directions. Based on theoretical analysis and experimental observations, it is concluded that small orientation errors $\left(<3^{\circ}\right)$ have no distinguishable effects on the estimation of energy expenditure. We propose an efficient method to compensate for larger orientation errors. The experimental results verified the effectiveness of this proposed compensation method.
\end{abstract}

\section{INTRODUCTION}

The standard reference for the measurement of physical activity is the metabolic energy expended due to that physical activity [1]. Consequently, a wide range of techniques for the measurement of physical activity have been developed, including direct observation, questionnaires and diaries, measurement of heart rate, oxygen uptake, determination of carbon dioxide production by the use of doubly labelled water, motion sensors and accelerometers [2]. Each technique has advantages and disadvantages. Accelerometry offers a practical and low cost method of objectively monitoring human movements, and has particular applicability to the monitoring of freeliving subjects, so it has been used to measure metabolic energy expenditure and to identify and classify movements performed by subjects. Many researchers who have investigated the validity of accelerometers as a tool for energy expenditure estimation in the activities of daily living reported favourably on the device [3] [4] [5] [6] [7]

For walking energy expenditure estimation, Ismail et al. [8] provided a prediction method based on the integration of the rectified force time curves from each measurement direction ( $r=0.73-0.92)$. Reswick et al. [9] applied a headmounted accelerometer to estimate walking energy expenditure on a large walkway. These results demonstrated that the integral of the modulus of accelerometer output was linearly related to the energy expenditure during walking, and this has led several researchers to hypothesize that the integral of the modulus of acceleration measured on the human body can be used to predict energy expenditure due to physical activity.

To evaluate the accuracy of energy expenditure, the effect of placement of accelerometers on the assessment of energy expenditure during walking has been studied [10]. However, another important issue, the effect of orientation error (the difference between the assumed orientations and the actually installed orientations of the sensitivity axes) has not yet been addressed.

The aim of this study was to explore the effect of orientation error of triaxial accelerometers on the assessment of energy expenditure. We provide a complete theoretical analysis of the effect of orientation error on the estimation of metabolic energy expenditure and suggest a simple but effective compensation method. The experimental results are provided to validate the theory.

\section{BACKGROUND}

\section{A. Metabolic Energy Expenditure}

There is a considerable body of research investigating the suitability of accelerometers to estimate energy expenditure, and determining the nature of the relationship between the measured accelerations and the actual metabolic energy expenditure [11]. Bouten et al [12] found that the best estimator across a range of daily activities was the sum of the integrals of the magnitudes of each of the three acceleration $(r=0.95, \quad P<0.001)$. The actual energy expenditure was then calculated using a linear regression.

$$
\begin{aligned}
& I A A_{\text {tot }}=\int_{t=0}^{T} A A_{t o t} d t=\int_{t=0}^{T}\left(\left|a_{x}\right|+\left|a_{y}\right|+\left|a_{z}\right|\right) d t \\
& E E_{\text {act }}=0.104+0.023 I A A_{\text {tot }}
\end{aligned}
$$

where

IAA $_{\text {tot }}$ is the sum of the integrals of the absolute value of accelerometer output from all the three measurement directions, $\mathrm{EE}_{\mathrm{act}}$ is the actual energy expenditure due to activity, $\mathrm{a}_{\mathrm{x}}, \mathrm{a}_{\mathrm{y}}$ and $\mathrm{a}_{\mathrm{z}}$ are accelerometer output from all the three measurement directions respectively, and $\mathrm{T}$ is integration period.

\section{B. Orientation Error Analysis and Compensation Method}


The orientation error is defined as the difference between the desired sensitivity axis and the actually sensitivity axis. See Fig. 1, where the orientation error can be determined by using three parameters (e.g. three Euler angle, $\alpha, \beta, \gamma$, where $\alpha$ is the rotation about the $\mathrm{Z}$ axis of the initial coordinate system, $\beta$ is the rotation about the $\mathrm{Y}$ axis of the newly generated coordinate system, followed by a rotation by $\gamma$ about the new $\mathrm{Z}$ axis [13]). For a Triaxial Accelerometer, because there is no gyroscope to provide direction information and the absolute directions of $\mathrm{X}, \mathrm{Y}$ and $\mathrm{Z}$ are not physically defined, the estimation of the three Euler angles is impossible.

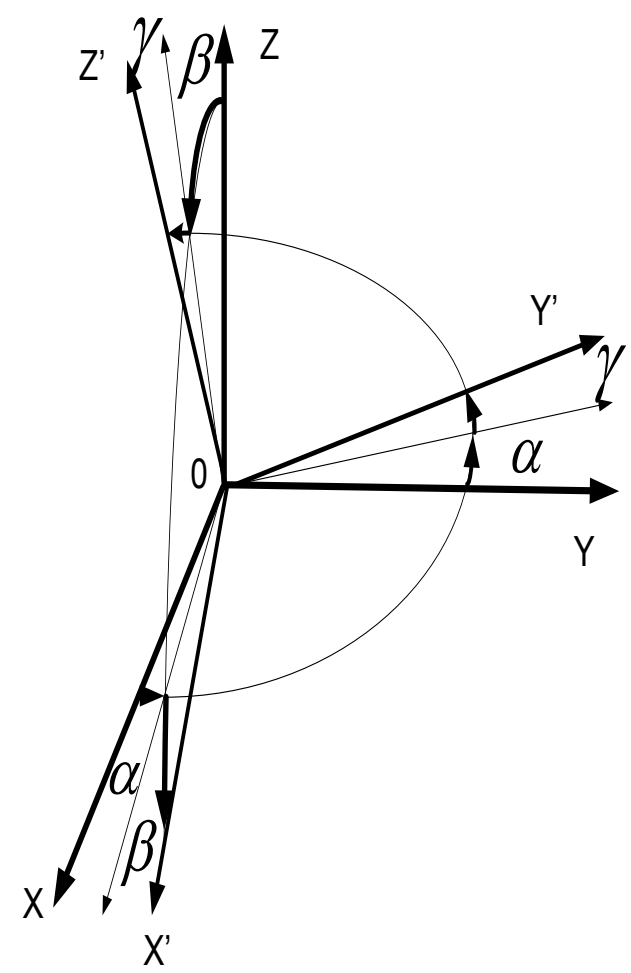

Fig. 1. Euler angles

From equation (2), we know that for the estimation of energy expenditure, it is important to get a reliable value of $I A A_{\text {tot }}$. Equation (1) implies that by analysing the values of $A A_{\text {tot }}$ under different orientation directions, the effects of orientation error on the assessment of energy expenditure can be achieved. Next, we show that for a given acceleration $A$, its corresponding $A A_{\text {tot }}$ is different under different orientation. Based on this observation, a simple but effective method is presented to compensate orientation error.

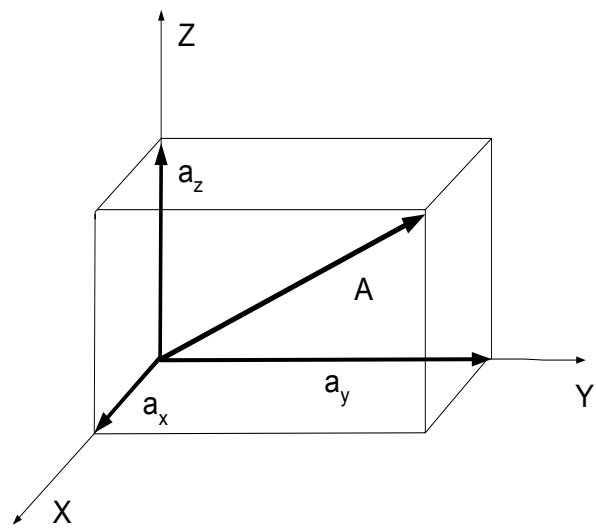

Fig. 2. The projections of acceleration A.

See Fig. 2, where $\boldsymbol{A}$ is the acceleration to be measured, and $a_{x}, a_{y}$ and $a_{z}$ are its projections on three sensitivity axes respectively. Then, we have

$$
\begin{aligned}
& A A_{\text {tot }}=\left|a_{x}\right|+\left|a_{y}\right|+\left|a_{z}\right| \\
& |A|=\sqrt{a_{x}^{2}+a_{y}^{2}+a_{z}^{2}}
\end{aligned}
$$

If we assume a Triaxial Accelerometer is an amplifier, and define $\kappa=\frac{A A_{t o t}}{|A|}$ as its gain, then $\kappa$ can be calculated from $a_{x}, a_{y}$ and $a_{z}$ :

$$
\kappa=\frac{A A_{t o t}}{|A|}=\frac{\left|a_{x}\right|+\left|a_{y}\right|+\left|a_{z}\right|}{\sqrt{a_{x}^{2}+a_{y}^{2}+a_{z}^{2}}} .
$$

It can be verified by the Lagrange function that $\kappa$ is in the range of 1 to $\sqrt{3}$.

When an orientation error exists, the projections of a given acceleration will be changed. Assuming the projections of acceleration A with a certain orientation error are $\bar{a}_{x}$,

$\bar{a}_{y}$ and $\bar{a}_{z}$, then

$$
\begin{aligned}
& \overline{A A}_{\text {tot }}=\left|\bar{a}_{x}\right|+\left|\bar{a}_{y}\right|+\left|\bar{a}_{z}\right|=\bar{\kappa}|A|=\frac{\bar{\kappa}}{\kappa} A A_{\text {tot }}, \text { or } \\
& A A_{\text {tot }}=\frac{\kappa}{\bar{\kappa}} \overline{A A_{\text {tot }}}
\end{aligned}
$$

Where

$$
\bar{\kappa}=\frac{\overline{A A}_{t o t}}{|A|}=\frac{\left|\bar{a}_{x}\right|+\left|\bar{a}_{y}\right|+\left|\bar{a}_{z}\right|}{\sqrt{a_{x}^{2}+a_{y}^{2}+a_{z}^{2}}} \in[1, \sqrt{3}] .
$$

Now, it is clear that equation (4) can be used to compensate $A A_{\text {tot }}$ for the orientation error. 


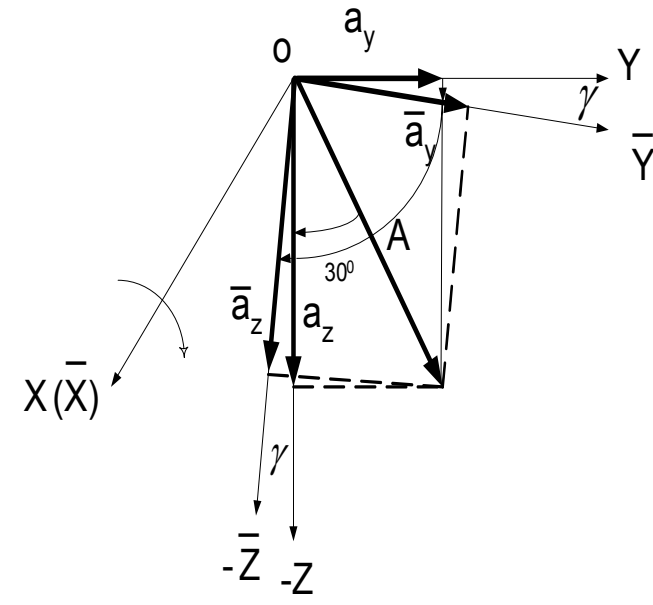

Fig. 3. A small orientation error

When the orientation error is small, its effects on the energy expenditure can be neglected. For example, when $\alpha=0^{\circ}, \beta=0^{0}, \gamma \leq 3^{0}$, the measured acceleration $\mathrm{A}$ is in the Z-O-Y plane and has a 30 degree angle with $\mathrm{Z}$ axis (see Fig. 3), then the relative error is only:

$$
\left|\frac{\overline{A A}_{t o t}-A A_{t o t}}{A A_{t o t}}\right| \times 100 \%<1.3 \%
$$

Similarly, for additional orientation error in the other two axes ( $\mathrm{Y}$ and $\mathrm{Z}$ ), i.e. $\alpha \leq 3^{\circ}, \beta \leq 3^{0}, \gamma \leq 3^{0}$, the cumulative effect of the orientation error is less than $5 \%$.

\section{EXPERIMENTAL VERIFICATION}

\section{A. Subjects}

The subjects were 4 healthy volunteers (two females, two males) aged (mean \pm SD) $30 \pm 1.5$ years, body weight: 63.5 $\pm 5.6 \mathrm{~kg}$, height: $174.0 \pm 6.8 \mathrm{~cm}$.

\section{B. Procedure}

Each subject walked on the treadmill for 30 minutes. His/her preferred walking speed was defined by progressively increasing the treadmill speed from $3 \mathrm{~km} . \mathrm{h}^{-1}$ to $6 \mathrm{~km} . \mathrm{h}^{-1}$. They were asked to state which speed was the most comfortable for them. The preferred speed for man was $5 \mathrm{~km} \cdot \mathrm{h}^{-1}$, while for woman the preferred speed was $4 \mathrm{~km} \cdot \mathrm{h}^{-1}$. After a resting period, the subjects walked at two speeds, which were $4 \mathrm{~km} \cdot \mathrm{h}^{-1}$ and $5 \mathrm{~km} . \mathrm{h}^{-1}$. For each speed, a triaxial accelerometer was first attached to the lower back for 5 minutes at the correct orientation to record body accelerations without orientation error. Then the triaxial accelerometer was attached to the lower back at arbitrary orientation for 5 minutes. After a second resting period, the triaxial accelerometer was reattached to the lower back at another arbitrary orientations to record body accelerations for a further 5 minutes. We repeated this procedure for 15 times for each subject. The speed was then changed and the above procedure was retested for the new speed.

The gait style was assumed to be constant during each 5 minutes exercise because the subjects were well trained at treadmill walking through the preferred speeds, and that the accelerometer output would not vary from one step to another.

Only the last 2 minutes of each exercise was used for the data analysis. It was assumed that 3 minutes warm up was sufficient to reach a steady state of energy expenditure.

\section{Accelerometer}

The accelerometer was designed in Biomedical System Lab (School of Electrical Engineering and Telecommunication, UNSW), and it consisted of two orthogonally mounted bi-axial piezo-resistive ADXL210 devices enclosed in a small pager casing. Telemetry was used to transmit the data to a personal computer where the data was stored [14]. An anti-aliasing analogue low-pass filter at $50 \mathrm{~Hz}$ was built in the device to reduce the bandwidth.

\section{Data Reductions}

Before further analysis, a digital high pass filter was applied to the raw signal of the accelerometer output $\left(f_{c}=\right.$ $0.11 \mathrm{~Hz}$ ) in order to remove the DC value (i.e. the gravity influence). Also the signal was passed through a low pass filter $\left(f_{c}=20 \mathrm{~Hz}\right)$ to subtract high frequency noise that is not expected to arise from human movement. The filtered data is analysed by using the calculation from equation (1) to (4), where the integration period $\mathrm{T}$ is 60 seconds.

\section{RESULT}

To test the efficiency of the proposed compensation technique (equation (4)), we take the difference for each subject between the 'true' value of the energy estimation, and both the uncompensated and compensated values. These are then analysed statistically to find the root mean square error (RMS). These results are summarised in table I. From this, we see the significant improvement (20\% - 59\%) in the proposed compensation technique in estimation of the energy expenditure. We also note that the improvement at the higher speed $\left(5 \mathrm{~km} \cdot \mathrm{h}^{-1}\right)$ is greater than that at the lower speed, implying that this compensation is more critical with the increased velocity. Indeed, the RMS (compensated) of the $5 \mathrm{~km} \cdot \mathrm{h}^{-1}$ data is significantly the same as that for the RMS of the $4 \mathrm{kmh}^{-1}$ data, implying that the technique is capable of estimating the energy expenditure to the same level of precision, no matter what the walking speed.

This is also demonstrated from the graphs in Fig.4, where we present the results for just two subjects. It is clear that the errors for compensated estimates are very much less than those for the uncompensated estimates, and also that the estimation errors increase with the increased speed (cf. $4 \mathrm{kmh}^{-1}$ and $5 \mathrm{~km} \cdot \mathrm{h}^{-1}$ ). 
TABLE I

The root mean square error for Energy Expenditure estimation $\left(\mathrm{J} \mathrm{min}^{-1} \mathrm{~kg}^{-1}\right)$

\begin{tabular}{|c|c|c|c|}
\hline \multirow[b]{2}{*}{ Subject } & \multicolumn{3}{|c|}{$4 \mathrm{kmh}^{-1}$} \\
\hline & Uncompensated & Compensated & $\begin{array}{c}\text { Improvement } \\
(\%)\end{array}$ \\
\hline Male $_{1}$ & 0.1859 & 0.1488 & 20.0 \\
\hline $\mathrm{Male}_{2}$ & 0.1841 & 0.155 & 15.8 \\
\hline Female $_{1}$ & 0.2537 & 0.1875 & 26.1 \\
\hline Female $_{2}$ & 0.3374 & 0.2658 & 21.2 \\
\hline \multirow[b]{2}{*}{ Subject } & \multicolumn{3}{|c|}{$5 \mathrm{~km} \cdot \mathrm{h}^{-1}$} \\
\hline & Uncompensated & Compensated & $\begin{array}{c}\text { Improvement } \\
(\%)\end{array}$ \\
\hline Male $_{1}$ & 0.2804 & 0.115 & 59.0 \\
\hline $\mathrm{Male}_{2}$ & 0.3075 & 0.1295 & 57.9 \\
\hline Female $_{1}$ & 0.4867 & 0.232 & 52.3 \\
\hline Female $_{2}$ & 0.4519 & 0.2012 & 55.5 \\
\hline
\end{tabular}

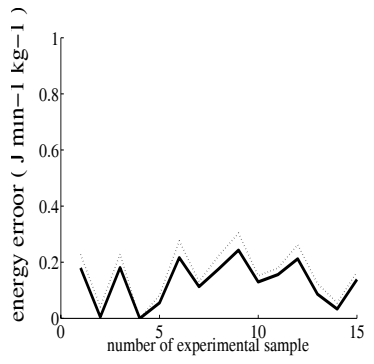

(a) Male with speed $4 \mathrm{~km} \cdot \mathrm{h}-1$

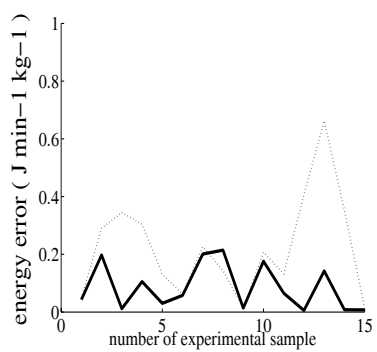

(c) Male with speed $5 \mathrm{~km} . \mathrm{h}-1$

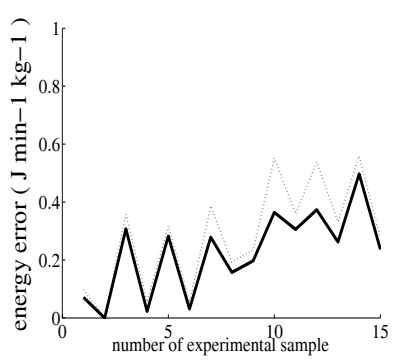

(b) Female with speed $4 \mathrm{~km} . \mathrm{h}-1$

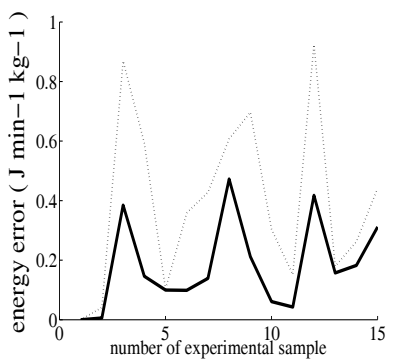

(d) Female with speed $5 \mathrm{~km} . \mathrm{h}-1$
Fig. 4. The errors of the energy expenditure estimation with compensation (solid line) and without compensation (dotted line).

\section{CONCLUSION}

This study explores the orientation error of triaxial accelerometer on the assessment of energy expenditure. There are two major contributions in this study. Firstly, it is found that small orientation errors $\left(<3^{\circ}\right)$ have no distinguishable effect on the estimation of energy expenditure. Secondly, the compensation method proposed has shown a possible improvement (table I) between $20 \%$ and $59 \%$ in the estimation of energy expenditure, and at least for this study it has proved to be as effective for the higher velocity as that for the lower. This could be verified by expanding the number of subjects and the range of speed.

\section{ACKNOWLEDGMENT}

We would like to acknowledge the valuable financial support from the Australian Research Council (ARC), the ARC grant number is DP0452186.

\section{REFERENCES}

[1] H.J. Montoye, R. Washburn, S. Servais, A. Ertl, J.G. Webster, and F.J. Nagle. "Estimation of energy expenditure by a portable accelerometer". Med. Sci. Sports Exerc. Vol. 15(5), pp. 403-407,1983.

[2] An analysis of research on preventing falls and falls injury in older people: community settings. Technique report, National Ageing Research Institute, for the Commonwealth Department of Health and Aged Care, 2000.

[3 ] G. Currie, D. Rafferty, G. Duncan, F. Bell, and A. L. Evans, "Measurement of gait by accelerometer and walkway: A comparison study," Med. Biol. Eng. Comp. vol. 30, pp. 669-670, 1992.

[4] J. R. W. Morris, "Accelerometry-A technique for the measurement of human body movements," J. Biomech, vol. 6, pp. 729-736, 1973.

[5] J. D. Frost, "Triaxial vector accelerometry: A new method for quantifying tremor and ataxia," IEEE Trans. Biomed. Eng. vol. BME-25, pp.17-27, 1978 .

[6] M. Salzer, "Three-dimensional tremor measurements of the hand," $J$. Biomech. vol. 5, pp. 217-221, 1972.

[7] C.V. Bouten, K.R. Westerterp, M. Verduin, and J.D. Janssen, "Assessment of energy expenditure for physical activity using a triaxial accelerometer," Med. Sci. Sports Exerc. vol. 26, pp. 1516-1523, 1994.

[8] A. H. Ismail, J. W. Baraby, and C. B. Smith, "Relationships between mechanical force and physiological cost during gait in adult men," in Biomechanics: Proceeding of the C.I.C. Symposium Biomechanics, J. M. Cooper, Ed. Chicago: The Athlete Inst. pp. 99-106,1971.

[9] J. Reswick, J. Perry, D. Antonelli, N. Su, and C. Freeborn, "Preliminary evaluation of the vertical acceleration gait analyzer (VAGA)," in Proc. 6th Annu. Symp. External Control Extremities, Dubrovnik, pp.305-314, 1978

[10] C.V. Bouten, A.A. Sauren, M. Verduin, and J.D. Janssen. "Effects of placement and orientation of body-fixed accelerometers on the assessment of energy expenditure during walking", Med. Biol. Eng. Comp. Vol. 35(1), pp. 50-56, 1997.

[11] C.V. Bouten, K.T. Koekkoek, M. Verduin,, R. Kodde, and J.D. Janssen, "A triaxial accelerometer and portable data processing unit for the assessment of daily activity", IEEE Transactions on Biomedical Engineering, vol. 44(3), pp. 136-147, 1997.

[12 ] C.V. Bouten, K.R. Westerterp, M. Verduin and J.D. Janssen, "Assessment of energy expenditure for physical activity using a triaxial accelerometer," Med. Sci. Sports Exerc. vol. 26, pp. 1516-1523, 1994. [13] M.E. Rose, Elementary Theory of Angular Momentum, Wiley: New York 1957

[14] M.J. Mathie, A.C.F. Coster, N.H. Lovell and B.G. Celler, "Detection of daily physical activities using a triaxial accelerometer", Med. Biol. Eng. Comp. vol. 41(3), pp.296 - 301, 2003. 